\title{
Nonlinear effects in life sciences
}

\author{
Tomasz Kapitaniak ${ }^{1, a}$ and Sajad Jafari ${ }^{2}$ \\ ${ }^{1}$ Division of Dynamics, Lodz University of Technology, Stefanowskiego 1/15, \\ 90-924 Lodz, Poland \\ ${ }^{2}$ Biomedical Engineering Department, Amirkabir University of Technology, \\ Tehran 15875-4413, Iran
}

Received 20 June 2018

Published online 19 October 2018

\begin{abstract}
This topical issue collects contributions of recent achievements and scientific progress related to the connection between nonlinear dynamics and life sciences. The individual papers focus on different questions of present day interest in both theoretical and applied aspects of nonlinear dynamics in applied to life sciences.
\end{abstract}

All biological systems can be classified as open, dissipative and non-linear. Mathematical knowledge has been used to describe, predict and control the phenomena observed in a number of fundamental systems leading to the development of mathematical biology which relies to a large extent on the approaches of non-linear dynamics, e.g. [1-9].

Nowadays there is growing evidence that future research on neural systems and higher brain functions will require a combination of classic neuroscience and the more recent nonlinear dynamics. The neuronal system composed of neurons and gliocytes is often sensitive to external forcing and internal shift in functional parameters, so that the appropriate response can be selected. This characteristic resembles the properties of chaotic systems. There is some evidence to support the claim that chaos occurs in many biological systems, like the human brain and heart. For example, it appears that the dynamics in electroencephalogram (EEG) signals are chaotic. The EEG signals may look random to outside observers, but there are hidden patterns in their randomlike appearance. Another example of chaos in biological systems is the dynamics at the neuronal level (cellular and subcellular). The transportation and storing of information in the brain is believed to be accomplished by the impulse trains produced by neurons. These trains of impulses or action potentials are often organized as sequences of bursts. The most important information in these sequences involves their temporal patterns, which are also known as interspike intervals (ISIs). The ISIs in a single neuron can show different behaviors including chaos. These impulses are generated by the interaction between the gating of ion channels and the axon's membrane voltage. Hodgkin and Huxley were the pioneers who proposed a dynamical system model of this interaction that predicted realistic action potentials.

Their model has been simplified in several forms by some other researchers. There are many recent papers that clearly show the existence of well known bifurcations and routes to chaos in heart. Also similar claims exist about kidney, lung, ... More than that, many diseases and disorders have been named dynamical diseases. From them we can point out migraine, epilepsy, bipolar disorder, and so on.

a e-mail: Tomasz.Kapitaniak@p.lodz.pl 
This special issue reviews the current state of the art in the research on life science from the viewpoint of nonlinear dynamics and point out the directions of further studies.

The issue starts with four mini reviews. Some papers are about nonlinear diseases and disorders. The authors of paper [10] try to answer the question "Does the onset of epileptic seizure start from a bifurcation point?". In paper [11], we try to propose a control method for HIV infection. General review on the monostability, bistability, periodicity and chaos in gene regulatory network is presented in paper [12]. Chaos control in enzyme-substrate reaction system using recursive backstepping sliding mode control, has been done by the authors of paper [13]. Next three papers investigate different dynamical models of a single neuron [14-16]. Paper [14] considers the model based on the Hodgkin-Huxley formalism with bistability between a silent state, which is a rare attractor and oscillatory bursting attractor, the authors demonstrate that the noise-induced switching between these two stable attractors depends on the structure of the phase space and the disposition of the coexisting attractors to each other. In paper [15], based on an improved Hodgkin-Huxley neuron model (driven by the electromagnetic induction), the effects of temperature and electromagnetic induction on the action potential of neuron has been investigated by numerical computations. Based on FitzHugh-Nagumo neuron model and by calculating Fourier coefficients for quantitatively characterizing the efficiency of the signal transmission, the authors in paper [16] investigate the effects of autapse with constant or time-periodic coupling intensity on signal transmission.

Some other researches investigate different phenomena in the networks of neurons. In paper [17], spatial pattern transitions have been simulated in a two-dimensional neuronal network composed of excitatory coupled neurons with autapse which can induce excitability transition. The authors of paper [18] have designed a network of three coupled neurons. They show that such a network can have multiple coexisting attractors including chaotic strange attractors. Based on an improved HR neuron model, the effects of electrical and chemical autapses on the firing activities of single neurons have been studied by the authors in paper [19]. The wave propagation in a forward feedback neural network has been also discussed by considering autapstic regulation under different intensities of electromagnetic induction. The authors of paper [20] investigate twin attractors in a two-neuron-based non-autonomous Hopfield neural network (HNN) through numerical analyses and hardware experiments. Considering brain as the most complex network of neurons, the brain-like large scale cognitive networks and their dynamics have been investigated by the authors in paper [21].

Beside neurons and neuronal networks, the dynamics of other biological systems have been noticed by the authors in this special issue. The authors of paper [22] have investigated the synchronization phenomenon in a network of fractional-order pancreatic $\beta$-cells. Paper [23] investigates the dynamical properties of a fractional order coronary artery system and its control with uncertainties in the parameters. Using a recently developed model of NF-B regulation, paper [24] examines the collective dynamics of a network of NF-B oscillators that are coupled exogenously by a common drive. In paper [25], the authors have investigated this transition both from a psychophysics perspective and in the development of a dynamic model of the visual system based on a spatially coupled array of nonlinear damped mass-springs cells. They present the results of the experiments using rigidly moving arrays of dots of different levels of sparseness and differing displacement magnitudes. Paper [26] investigates the influence of non-Gaussian noise on a tumor growth system under immune surveillance. The authors of paper [27] present the connectivity patterns of evolving large scale epileptic networks. They have employed a cross-entropy measure in the frequency domain on EEG signals to infer the networks before and during episodes of epileptic seizures. 
Three papers focus on the analysis of nonlinear and complex real signals obtained from patients. In paper [28], extreme events are shown on electroencephalographic (EEG) recordings of rodents with induced ischemic stroke. These events represent themselves as post-stroke epileptic seizures in the form of spontaneous high-amplitude oscillations, which appear during the first 2-3 hours after induced focal cerebral ischemia. The authors of paper [29] have developed a low-cost and portable eightchannel EMG acquisition system which consists of the related analogue front end (AFE), main control unit (MCU) and power supply system. In paper [30], fractional fuzzy entropy algorithm has been designed by combing the concept of fractional information and fuzzy entropy algorithm. The complexity of chaotic signals and systems has been analyzed by this method.

Finally, last three papers investigate ecological systems. Paper [31] reports the emergence of spiral wave chimera patterns in locally coupled ecological network composed of diffusible prey-predator species. Dynamical transitions from spiral wave states to spiral wave chimera followed by incoherent dynamics with respect to increasing diffusion coefficients have been explained as well. In paper [32], the authors study the peculiarities of spiral attractors in Rosenzweig-MacArthur model, which relates to the life-science systems and describes the dynamics in a food chain "prey-predatorsuperpredator". The authors of paper [33], introduce and analyze a predator-prey model with Allee effect and seasonally forcing in the prey's growth rate.

Thus, this special issue provides a wide spectrum of current research on nonlinear dynamics in biology and life science and we hope that the related researchers in this field will find it useful. We wish to express our appreciation to the authors of all the papers in this special issue for the excellent contributions as well as to many reviewers for their high-quality work on reviewing the manuscripts.

\section{References}

1. I. Prigogine, G. Nicolis, A. Babloyants, Phys. Today 25, 23 (1972)

2. J.D. Murray, Mathematical biology, I. An introduction (Springer, Berlin, 2003)

3. J.D. Murray, Mathematical biology, II. Spatial models and biomedical applications (Springer, Berlin, 2003)

4. A.T. Winfree, The geometry of biological time (Springer, Berlin, 1980)

5. A. Golberger, Biochemical oscillations and biological rhythms (Cambridge University Press, Cambridge, 1996)

6. A. Stefanovska, Contemp. Phys. 40, 31 (1999)

7. L. Glass, M.C. Mackey, From clocks to chaos: the rhythms of life (Princeton University Press, Princeton, 1988)

8. N.B. Janson, Contemp. Phys. 53, 137 (2012)

9. M.S. El Naschie, T. Kapitaniak, Phys. Lett. A 147, 275 (1990)

10. F. Nazarimehr, S.M.R.H. Golpayegani, B. Hatel, Eur. Phys. J. Special Topics 227, 697 (2018)

11. H. Jahanshahi, Eur. Phys. J. Special Topics 227, 707 (2018)

12. Q. Lai, X.-W. Zhao, J.-N. Huang, V.-T. Pham, K. Rajagopal, Eur. Phys. J. Special Topics 227, 719 (2018)

13. P.P. Singh, Ksh.M. Singh, B.K. Roy, Eur. Phys. J. Special Topics 227, 731 (2018)

14. N. Stankevich, E. Mosekilde, A. Koseska, Eur. Phys. J. Special Topics 227, 747 (2018)

15. Y. Yao, J. Ma, Eur. Phys. J. Special Topics 227, 757 (2018)

16. L. Lu, B. Kirunda, Y. Xu, W. Kang, R. Ye, X. Zhan, Y. Lia, Eur. Phys. J. Special Topics 227, 767 (2018)

17. Q. Xu, Z. Song, H. Qian, M. Chen, P. Wu, B. Bao, Eur. Phys. J. Special Topics 227, $777(2018)$

18. F. Bertacchini, E. Bilotta, M.C. Lombardo, M. Sammartino, P. Pantano, Eur. Phys. J. Special Topics 227, 787 (2018) 
19. M. Ge, Y. Xu, Z. Zhang, Y. Peng, W. Kang, L. Yang, Y. Jia, Eur. Phys. J. Special Topics 227, 799 (2018)

20. K. Rajagopal, J.M. Munoz-Pacheco, V.-T. Pham, D.V. Hoang, F.E. Alsaadi, F.E. Alsaadi, Eur. Phys. J. Special Topics 227, 811 (2018)

21. Y. Li, B. Jia, X. Zhang, and Y. Yang, Eur. Phys. J. Special Topics 227, 821 (2018)

22. K. Rajagopal, V.-T. Pham, F.E. Alssadi, F.E. Alsaadi, A. Karthikeyan, P. Duraisamy, Eur. Phys. J. Special Topics 227, 837 (2018)

23. R. Donepudi, R. Ramaswamy, Eur. Phys. J. Special Topics 227, 851 (2018)

24. P. Celka, T. Hine, Eur. Phys. J. Special Topics 227, 865 (2018)

25. A.C. Rodrigues, L.O.S.F. Cabocio, H.A. Cerdeira, E. Amaro Jr. B.S. Machado, Eur. Phys. J. Special Topics 227, 883 (2018)

26. L. Du, Q. Guo, Z. Sun, Eur. Phys. J. Special Topics 227, 895 (2018)

27. E. Zambrano-Serrano, J.M. Munoz-Pacheco, L.C. Gomez-Pavon, A. Luis-Ramos, G. Chen, Eur. Phys. J. Special Topics 227, 907 (2018)

28. A.N. Pisarchik, V.V. Grubov, V.A. Maksimenko, A. Luttjohann, N.S. Frolov, C. Marques-Pascual, D. Gonzales-Nieto, M.V. Khramova, A.E. Hramov, Eur. Phys. J. Special Topics 227, 921 (2018)

29. C. Mi, T. Zhou, B. Wei, Y. Wang, L. Zou, Eur. Phys. J. Special Topics 227, 933 (2018)

30. S. He, K. Sun, R. Wang, Eur. Phys. J. Special Topics 227, 943 (2018)

31. Y.V. Bakhanova, A.O. Kazakov, A.G. Korotkov, T.A. Levanova, G.V. Osipov, Eur. Phys. J. Special Topics 227, 959 (2018)

32. A.B. Saad, O. Boubaker, Eur. Phys. J. Special Topics 227, 971 (2018)

33. S. Kundu, S. Majhi, P. Muruganandam, D. Ghosh, Eur. Phys. J. Special Topics 227, $983(2018)$ 